\title{
Cultural Transmission and Extortion
}

\author{
Garret Ridinger \\ Department of Managerial Sciences, College of Business, University of Nevada, Reno, 1664 N Virginia St., \\ Reno, NV 89557, USA; gridinger@unr.edu
}

Received: 23 May 2018; Accepted: 10 July 2018; Published: 15 July 2018

\begin{abstract}
This paper explores the role of cultural transmission in extortion. Extortion is modeled as an asymmetric contest where individuals from one group attempt to take from individuals in another group. Using a model of cultural transmission, this paper finds the existence of a unique asymptotically stable equilibrium where there are a fraction of people who defend against extortion and a fraction of people who take from others. The degree of extortion is decreased when: (1) extortion is less effective; (2) socialization efforts of parents who resist are more effective; and (3) socialization efforts of parents who abstain from extortion are more effective. A key finding is the existence of a complementarity between the effectiveness of socialization. When socialization is more effective for resisting extortion and choosing not to extort, there is a much larger reduction of extortion than either increase could achieve alone. This provides a potential explanation for why some countries like Singapore and Hong Kong were able to greatly reduce corruption and suggests potential policy applications.
\end{abstract}

Keywords: cultural transmission; evolutionary game theory; contests; extortion; corruption

\section{Introduction}

In all societies, there exist individuals that attempt to take from others. For example, in Aceh, as truck drivers travel their route, they are stopped at security checkpoints [1]. Soldiers stationed at the checkpoints are supposed to provide security. Instead, there are many who extort bribes. It is so common that most drivers automatically pay them, with many who refuse beaten until they do. In the Philippines in 1975, some tax authorities extorted money from citizens by threatening to falsely inflate their tax liability and forcing them to undergo costly litigation if they refused [2]. Both the soldiers and tax authorities have advantages through superior weapons or political connections that make it difficult for people to challenge their corrupt actions.

Extortion is a particularly damaging form of corruption. Attempts to fight corruption have been largely unsuccessful as countries that are corrupt typically remain so despite interventions. Anti-corruption programs that attempt to improve or add resources to legal and financial institutions have found little evidence of success [3]. Singapore and Hong Kong are two notable exceptions of countries that were able to greatly reduce corruption [3]. Both countries created independent anti-corruption agencies. In Hong Kong, an education program was implemented to try to change the culture of corruption [4]. In Singapore, pay was increased, public officials were rotated to prevent tie formation, rules were made publicly available, and rewards were given to people who refused bribes [3]. In Singapore and Hong Kong, the anti-corruption programs did lower the benefits and costs of corruption, but they also emphasized the moral importance of not engaging in corruption. Empirical evidence suggests that culture may have a strong effect on individual tendencies to engage in corruption [5-10]. Through the provision of education and information, it is possible that the success of Hong Kong and Singapore is in part due to changes in the distribution of cultural values.

This paper examines how culturally transmitted values and differences in power influence the persistence of extortion. This is addressed theoretically by analyzing an asymmetric contest in 
a model of cultural transmission [11-13]. In the model, there are two populations. One population (citizens) consists of people who are willing to fight against extortion and those who are not. The other population (elites) contains people who choose to extort from citizens and those who abstain. In the model, values are socially transmitted to children by their parents as well as by society. Parents choose costly socialization efforts that influence the probability that their child will have the same values as they do. The model introduces a new feature into the cultural transmission model by allowing for a bias in socialization efforts by parents that can differ depending on their type. This bias can capture how parental socialization may be more or less effective depending on the environment [14-16]. For example, a parental lesson that bribery is wrong may be re-enforced by what children learn in school, read in books, or watch on television. After socialization, citizens and elites are randomly matched and engage in the contest.

\subsection{Key Result}

The key result is the existence of a unique asymptotically stable equilibrium where there are a fraction of people who defend against extortion and a fraction of people who chose to take from others. When there are wide differences in power between those who extort and those who resist, extortion is quite prevalent. Extortion is decreased when: (1) the socialization efforts of parents who resist are more effective and (2) the socialization efforts of parents who abstain from extortion are more effective. In addition, there exists a complementarity between socialization efforts, showing that, when socialization is more effective for resisting extortion and abstaining from extortion, there is a much larger reduction of extortion than either increase could achieve alone. This provides a potential explanation for why Singapore and Hong Kong were able to greatly reduce corruption, and suggests that cultivating "good citizens" may lower the prevalence of extortion in a society by changing underlying preferences.

\subsection{Prior Literature}

In this paper, extortion is modeled as an asymmetric contest where individuals from one group attempt to take from individuals in another group. The study of extortion is related to a wider literature examining conflict. Introduced by Smith and Price [17], the hawk-dove game has been extensively used to gain insights into the evolution of conflict [18-24]. In the standard hawk-dove game, two players can compete over a shared resource. Players can choose the hawk strategy in which they fight for the resource or the dove strategy where they do not enter the conflict. A different approach is the producer-scrounger game that has been used to study the evolution of food stealing in animals [25-27]. In the game, animals can allocate time between producing food and scrounging food from others. If in possession of food, an animal can choose to defend against theft by scroungers. If not in possession of food, an animal can choose whether to take food from the producers. Broom et al. [27] found that, as the intensity of conflict increases, taking food from producers and not resisting if a scrounger takes their food becomes increasingly common strategy in the population. Both the hawk-dove and the producer-scrounger game capture important elements of conflict but differ from extortion in a few key ways. In both the hawk-dove game and the producer-scrounger game, each player has the ability to take from others. However, extortion differs in that there is an asymmetry in the game where only one party has the ability to extort, and the other party may try to resist. Extortion further differs from the producer-scrounger game since extortion often occurs between groups with different levels of power. As a result, the model in this paper captures situations such as soldiers' decisions to extort truck drivers in Aceh [1] and tax authorities threatening to inflate tax liabilities of citizens to extort money [2]. Due to their position of power, the soldiers and tax authorities do not typically have to resist extortion by citizens. This difference makes it important to study extortion in a two population game.

This paper uses a model of cultural evolution to analyze the dynamics of extortion. Mathematical modeling of cultural evolution began with the seminal works of Cavalli-Sforza and Feldman [28] and Boyd and Richerson [29]. Both Cavalli-Sforza and Feldman [28] and Boyd and 
Richerson [29] introduced evolutionary models to study the population dynamics of cultural traits. In Cavalli-Sforza and Feldman [28], the probability of acquiring a cultural trait was influenced through three potential channels: vertically from parents to child, obliquely from the parents' generation to the child, and horizontally from child to child. Boyd and Richerson [29] incorporated additional transmission rules such as frequency-dependence where the probability of acquiring a cultural trait is influenced by frequency of the cultural trait in the population and payoff-based learning where cultural traits that produce higher payoffs are more likely to be imitated by others. Adding to our understanding of cultural evolution, numerous studies have examined how these transmission rules and other forms of social learning influences the adoption of cultural traits [29-35].

In both Cavalli-Sforza and Feldman [28] and Boyd and Richerson [29], transmission rules are modeled exogenously. However, there is reason to believe that parents may hold preferences over certain cultural traits and choose strategies that influence the chance that their child adopts those preferred traits [13]. Extending the approach of Cavalli-Sforza and Feldman [28], Bisin and Verdier [11] endogenize the vertical transmission from parents to child by allowing parents to invest in a costly effort that influences the probability that direct socialization is successful. This endogenous socialization approach has been used to study a wide range of topics including: political ideology and voting [36], preferences for social status [37], cooperation norms [38] and coordination [39]. Bisin et al. [40] also use this approach to study cooperation in a one-shot symmetric prisoner's dilemma where individuals are of two types: cooperators or defectors. The authors show that, for certain parameter ranges, there can exist a heterogeneous population of both cooperators and defectors. In contrast to Bisin et al. [40], this paper studies extortion using a contest that is more closely related to prior studies examining evolutionary behavior in hawk-dove games [17,22] and producer-scrounger games [25-27]. Additionally, by analyzing an asymmetric game, this paper extends the cultural transmission model used in Bisin and Verdier [11] to two populations. This methodological approach can be used in future work to analyze how endogenous socialization influences cultural transmission in a wider range of games. Finally, this paper introduces a bias in the effectiveness of direct socialization efforts by parents. The efforts that parents undertake to influence the cultural trait adopted by their child may have different returns depending on the environment or the specific cultural trait.

In a related paper, Hauk and Saez-Marti [4] examined the intergenerational transmission of corruption. Their model includes an infinitely-lived principal-agent who chooses between two projects to offer individuals. One project gives the principle the highest potential payoff if the agent is honest, but the worst payoff if the agent is dishonest. Individuals can be of two types: those who feel guilty about being dishonest and those who do not. These types are transmitted intergenerationally by parents and society. Hauk and Saez-Marti [4] show that, since the principal is unsure of the type, this leads to either a separating or pooling equilibrium. Their result sheds light on how culture may influence investment by individuals or firms in countries that have high or low corruption. This paper adds to this literature by focusing on how culture and biases in socialization influences extortion. By studying the dynamics of two populations, those who can choose whether to extort and those who can choose whether to resist, this paper examines how culture affects both sides of corrupt interactions. In contrast to Hauk and Saez-Marti [4], the model predicts a unique stable equilibrium where corruption will always occur. Differences in culture and power can influence the level of corruption, but corruption and resistance will persist in the population.

\section{Basic Contest}

Suppose there are two populations of equal size. Individuals in population one (citizens) have some value $V$ that they can choose to defend against possible extortion. In population one, there are 
two types of citizens: Type-A's value exerting effort in a contest for $V$ and Type-B's do not. ${ }^{1}$ Let the fraction of Type-A's value be $q$ and Type-B's value be $1-q$. In population two (elites), there are two types of individuals: Type-C's value exerting effort in a contest for $V$ and Type-D's do not. Let the fraction of Type-C's be $p$ and Type-D's be $1-p$. Each citizen $i$ from population one is randomly matched with an elite $j$ from population two. Both $i$ and $j$ observe the type they are matched with and choose a costly effort level that influences the probability that they will receive the value $V$ in the contest. The contest is assumed to be an asymmetric Tullock [41] contest defined as follows:

$$
P_{i}\left(x_{i}, x_{j}\right)= \begin{cases}\frac{x_{i}}{x_{i}+\lambda x_{j}}, & \text { if } x_{i}>0 \text { or } x_{j}>0, \\ \frac{1}{1+\lambda}, & \text { o.w. }\end{cases}
$$

The parameter $\lambda>0$ captures the asymmetry between the two populations on the probability of winning the contest. The utilities for a given type and corresponding match are summarized in Table 1. The costs are a function of effort and the cost of effort is assumed to be the same for each $i$ and $j ; x_{i}$ and $x_{j}{ }^{2}$ The maximized utilities for each type and match are summarized in Table $2 .^{3}$

Table 1. Individual match and utility function.

\begin{tabular}{|c|c|c|}
\hline & Type-C's $\left(U_{j}^{C}\left(x_{i}, x_{j}\right)\right)$ & Type-D's $\left(u_{j}^{D}\left(x_{i}, x_{j}\right)\right)$ \\
\hline Type-A's $\left(U_{i}^{A}\left(x_{i}, x_{j}\right)\right)$ & $P_{i}\left(x_{i}, x_{j}\right) \cdot V-c_{i}\left(x_{i}\right) \quad P_{j}\left(x_{i}, x_{j}\right) \cdot V-c_{j}\left(x_{j}\right)$ & $\begin{aligned}-c_{i}\left(x_{i}\right) & \\
& -c_{j}\left(x_{j}\right)\end{aligned}$ \\
\hline Type-B's $\left(U_{i}^{B}\left(x_{i}, x_{j}\right)\right)$ & $-c_{i}\left(x_{i}\right) \quad V-c_{j}\left(x_{j}\right)$ & $V-c_{i}\left(x_{i}\right) \quad-c_{j}\left(x_{j}\right)$ \\
\hline
\end{tabular}

Table 2. Individual match and maximized utilities.

\begin{tabular}{lccc}
\hline & Type-C's $\left(U_{j}^{C}\left(x_{i}^{\star}, x_{j}^{\star}\right)\right)$ & Type-D's $\left(U_{j}^{D}\left(x_{i}^{\star}, x_{j}^{\star}\right)\right)$ \\
\hline Type-A's $\left(U_{i}^{A}\left(x_{i}^{\star}, x_{j}^{\star}\right)\right)$ & $\frac{V}{(1+\lambda)^{2}}$ & $\frac{\lambda^{2} V}{(1+\lambda)^{2}}$ & 0 \\
\hline Type-B's $\left(U_{i}^{B}\left(x_{i}^{\star}, x_{j}^{\star}\right)\right)$ & 0 & $V$ & 0 \\
\hline
\end{tabular}

\section{Cultural Transmission}

In order for a contest with positive efforts to occur, it is necessary that individuals perceive some benefit from the contest. Valuations in contests are often taken as given, but in reality they may be influenced by culturally transmitted values. To capture this, imagine that people live two periods in overlapping generations. Suppose that each person is born, socialized, and then plays the contest in period $t$. In period $t+1$, each person has a single child and can choose a socialization effort level $d_{i \epsilon\{A, B, C, D\}}$. Socialization efforts increase the chance that a child will be the same type as their parents. Parents in period $t+1$ want to maximize the expected utility of their child, but they do so with

1 The results of the model are similar if both Type-A's and Type-B's value exerting effort, but Type-A's place a greater value on winning the contest compared to Type-B's. As long as there is a difference in the valuation of the contest between the two types, the results will be similar. Norming Type-B's value to zero simplifies the presentation of the results.

2 Assuming different linear costs of efforts between the groups gives predictable results. For example in subsequent analysis, assuming costs are higher for individuals in population one leads to a higher equilibrium fraction $p$ and a lower equilibrium fraction $q$. The reverse holds as well assuming costs are higher for population two. As a result, similar costs were assumed for ease of exposition.

3 It is assumed that both Type-Cs and Type-Ds via their position of authority receive some fixed benefit or salary that is independent of their decision to extort from citizens in population one. This benefit for ease of notation is assumed to be zero, since, as long as this benefit is the same for both types, the results will be unchanged. 
altruistic paternalism [11]. As a result, they use their own utility function to evaluate the utility of a child's potential type. These costly effort choices of parents influence the population dynamics of types.

Type-A parents can directly socialize their child to be type-A with probability $\theta d_{A}$, where $\theta>0$. If direct socialization fails, then the child undergoes oblique socialization. Oblique socialization is non-direct socialization that occurs from the parent's generation to the child's. That is, with probability $1-\theta d_{A}$, the child randomly selects another adult in the population to imitate. The term $\theta$ captures the effectiveness of a parents socialization efforts. As a result, the probability that a type-A parent will have a type-A child is $P^{A A}=\theta d_{A}+\left(1-\theta d_{A}\right) \cdot q$ and the probability the child will be type-B is $P^{A B}=\left(1-\theta d_{A}\right)(1-q)$. Similarly, a type-B parent will have a type-B child with probability $P^{B B}=d_{B}+\left(1-d_{B}\right)(1-q)$ and a type-A child with probability $P^{B A}=\left(1-d_{B}\right) \cdot q$.

Parents want to maximize the expected utility of their child based on the potential payoffs from playing contest. Define $V^{A A}$ as the utility that a type-A parent believes a type-A child will receive and $V^{A B}$ as the utility that a type-A parent believes a type-B child will receive. Here, $V^{A A}=U_{i}^{A}\left(x_{i}, x_{j}\right)$ and $V^{A B}=U_{i}^{A}\left(x_{i}, x_{j}\right)$. A type-A parent uses their own utility function to evaluate the potential utility of their children. Similarly, a type-B parent as $V^{B B}$ and $V^{B A}$.

A type-A parent faces the following maximization problem:

$$
\max _{d_{A} \in[0,1]} P^{A A} \cdot V^{A A}+P^{A B} \cdot V^{A B}-C\left(d_{A}\right) .
$$

Here, $C\left(d_{A}\right)$ is the cost of socialization effort. Following Montgomery [12] and Bisin and Verdier [13], for simplicity, assume that $C\left(d_{A}\right)=\frac{1}{2}\left(d_{A}\right)^{2}$. Solving for the optimal socialization effort gives $d_{A}^{\star}=\theta(1-q)\left[\triangle V^{A}\right]$ where $\triangle V^{A}=V^{A A}-V^{A B}$. Similarly, for type-B, we have $d_{B}^{\star}=q\left(\triangle V^{B}\right)$ where $\triangle V^{B}=V^{B B}-V^{B A}$. Both $\triangle V^{A}$ and $\triangle V^{B}$ can be viewed as measures of cultural distaste.

The change in $q$ from period $t$ to $t+1$ is as follows:

$$
q_{t+1}-q_{t}=-q \cdot P^{A B}+(1-q) \cdot P^{B A} .
$$

Substituting and rearranging gives:

$$
q_{t+1}-q_{t}=q(1-q)\left[\theta d_{A}^{\star}-d_{B}^{\star}\right]
$$

Taking the continuous time approximation and subbing in both $d_{A}^{\star}$ and $d_{B}^{\star}$ gives the replicator dynamic [12,13]:

$$
\dot{q}=q(1-q)\left[\theta^{2}(1-q)\left(\triangle V^{A}\right)-q\left(\triangle V^{B}\right)\right] .
$$

Derivation for population two is similar with Type-C's choosing socialization effort $d_{C}$ and type-D's choosing socialization effort $\beta d_{D}$ where $\beta>0$. Defining $\triangle V^{C}=V^{C C}-V^{C D}$ and $\triangle V^{D}=$ $V^{D D}-V^{D C}$, the replicator dynamic can be written as:

$$
\dot{p}=p(1-p)\left[(1-p)\left(\triangle V^{C}\right)-\beta^{2} p\left(\triangle V^{D}\right)\right] .
$$

\section{Contest with Cultural Transmission}

Using Table 2 and substituting the cultural distastes into the dynamics for $q$ and $p$, we have the following set of differential equations: ${ }^{4}$

$$
\dot{q}=q(1-q)\left[\theta^{2}(1-q)\left(\frac{p V}{(1+\lambda)^{2}}\right)-q\left(\frac{p \lambda V}{(1+\lambda)^{2}}\right)\right]
$$

4 See Appendix A for details. 


$$
\dot{p}=p(1-p)\left[(1-p)\left(\frac{q \lambda^{2} V}{(1+\lambda)^{2}}+(1-q) \cdot V\right)-\beta^{2} p\left(\frac{q \lambda V}{(1+\lambda)^{2}}\right)\right] .
$$

There exist a total of seven equilibria denoted as $\left(q^{\star}, p^{\star}\right)^{5}$ The four exterior equilibria on the corners are: $(0,0),(0,1),(1,0)$, and $(1,1)$. Two additional equilibria exist on the boundaries, namely $\left(\frac{\theta^{2}}{\theta^{2}+\lambda}, 1\right)$ and $\left(1, \frac{\lambda}{\beta^{2}+\lambda}\right)$. The interior equilibrium is $\left(\frac{\theta^{2}}{\theta^{2}+\lambda}, \frac{\theta^{2} \lambda+(1+\lambda)^{2}}{\theta^{2} \lambda+(1+\lambda)^{2}+\theta^{2} \beta^{2}}\right)$.

Proposition 1. The equilibrium $q^{\star}=\frac{\theta^{2}}{\theta^{2}+\lambda}$ and $p^{\star}=\frac{\theta^{2} \lambda+(1+\lambda)^{2}}{\theta^{2} \lambda+(1+\lambda)^{2}+\theta^{2} \beta^{2}}$ is the unique asymptotically stable equilibrium.

Proof. Please see Appendix A.

Figure 1 illustrates an example of the different equilibria that can occur in the system. The interior equilibrium is uniquely stable, showing that there will always be some fraction of individuals who extort and a fraction of individuals who resist. The equilibrium fraction of people willing to defend themselves against the aggressors is increasing in $\theta$. This suggests that, when socialization is more effective for parents of Type-A, there will be a larger frequency of resistance in the population. However, the fraction of Type-As is decreasing in $\lambda$. When Type-As are less effective at winning the contest with a Type-C, there will be less resistance in the population. The equilibrium level of Type-Cs is increasing in $\lambda$, and decreasing in $\theta$ and $\beta$. Interestingly, there is a complementarity between the effectiveness of socialization on the equilibrium level of Type-Cs. The term $\theta^{2} \beta^{2}$ suggests that the effectiveness of socialization for Type-As and Type-Ds can have a combined effect of lowering the level of extortion. This is illustrated in Figure 2, which plots the equilibrium levels of $p$ and $q$ as a function of $\lambda$. We can see that, as $\lambda$ increases, there is a large amount of extortion. Increasing the effectiveness of $\theta$ or $\beta$ does help, but the effect in reducing extortion is much larger when both $\theta$ and $\beta$ increase.

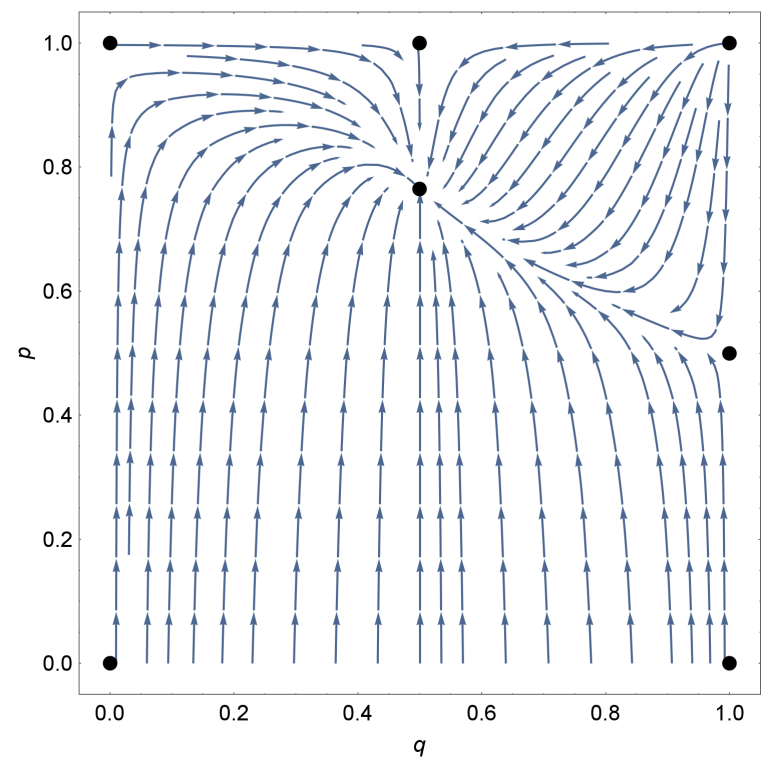

Figure 1. Example of phase plane $\left(\theta^{2}=2, \beta^{2}=2, \lambda=2, V=1\right)$.

5 If $p=0$, then any $q$ can be an equilibrium. In this case, there is no extortion so there is no payoff difference between type A's and B's. 

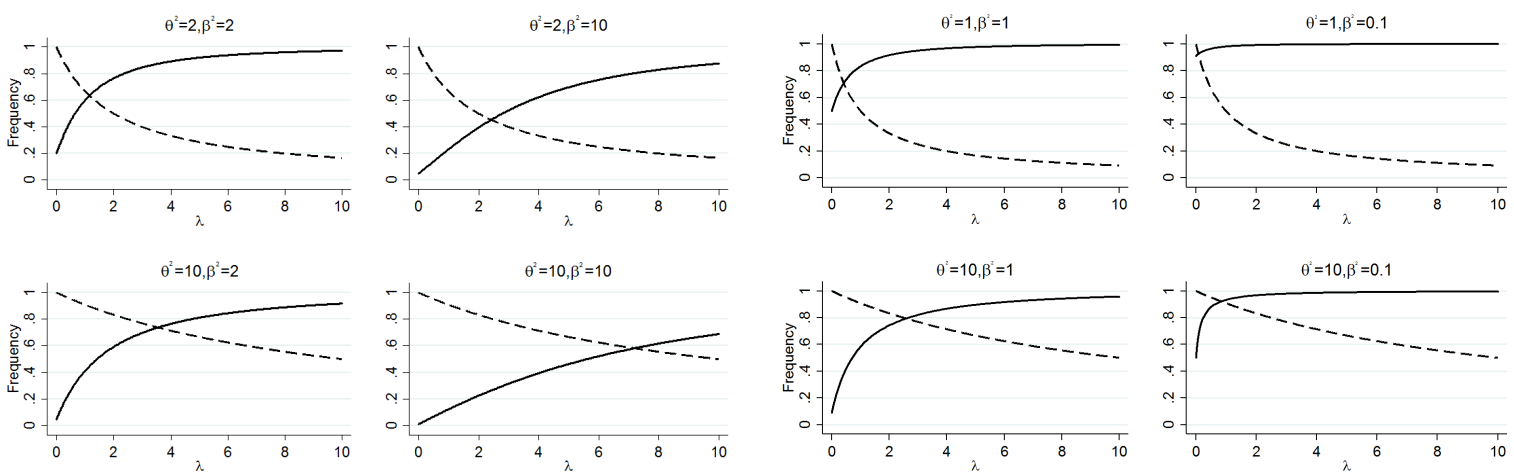

$\longrightarrow p-----q$

(a)

(b)

Figure 2. Changes in population frequencies as a function of $\lambda$. Subfigures (a) and (b) assume different values for the bias in socialization parameters: $\theta$ and $\beta$.

The biases in socialization efforts, $\theta$ and $\beta$, capture important features in influencing the frequency of types. When parents attempt to socialize their children, these efforts can be more or less effective depending on the environment in which the child is raised. For example, Type-Ds may teach their children that it is wrong to take from others. This lesson may be reinforced by the outside world. As a result, this lesson is more likely to lead children in a position of power to resist taking from others. Alternatively, even if Type-Ds teach their children that it is wrong to take from others, the children may receive the opposite lesson from the environment. This reduction in the effectiveness of parental effort leads to more extortion in the population. This suggests a potential reason for why issues like corruption and crime can be so persistent.

\section{Conclusions}

This paper introduces an asymmetric Tullock [41] contest into a model of cultural transmission to study the prevalence of extortion. The model introduces a new feature that allows for a bias in the socialization efforts by parents that can differ depending on their type. The key result is the existence of a unique stable equilibrium where there are a fraction of people who defend against extortion and a fraction of people who choose to take from others. An important finding is that there exists a complementarity between the effectiveness of socialization. When socialization is more effective for resisting extortion and abstaining from extortion, there is a much larger reduction of extortion than either increase could achieve alone. This result suggests an additional explanation for why some countries like Singapore and Hong Kong were able to greatly reduce corruption. This complementarity suggests that anti-corruption programs may be more effective by targeting corruption at both the top and bottom. This paper presents a step forward in trying to understand how cultural transmission can influence adversarial situations. Future research should explore the influence of other socialization and cultural factors such as conformity [35], identity formation [42], and beliefs [43].

Acknowledgments: I would like to thank Anne Carpenter, Stergios Skaperdas, Jean-Paul Carvalho, and two anonymous referees for their helpful comments and advice.

Conflicts of Interest: The author declares no conflict of interest.

\section{Appendix A}

Using the results from Table 2, we can calculate the cultural distaste parameters. Using the parents own utility function to calculate the cultural distastes of each parent, we have: 


$$
\begin{aligned}
\Delta V^{A} & =\left[p\left(\frac{V}{(1+\lambda)^{2}}\right)+(1-p)(V)\right]-[p(0)+(1-p)(V)] \\
\Delta V^{B} & =[p(0)+(1-p)(V)]-\left[p\left(\frac{-\lambda V}{(1+\lambda)^{2}}\right)+(1-p)(V)\right], \\
\Delta V^{C} & =\left[q\left(\frac{\lambda^{2} V}{(1+\lambda)^{2}}\right)+(1-q)(V)\right]-[q(0)+(1-q)(0)], \\
\Delta V^{D} & =[q(0)+(1-q)(0)]-\left[q\left(\frac{-\lambda V}{(1+\lambda)^{2}}\right)+(1-q)(0)\right] .
\end{aligned}
$$

Substituting the above cultural distaste parameters into the dynamics, Equations (1) and (2) give the differential equations in Section 4.

Proposition A1. The equilibrium $q^{\star}=\frac{\theta^{2}}{\theta^{2}+\lambda}$ and $p^{\star}=\frac{\theta^{2} \lambda+(1+\lambda)^{2}}{\theta^{2} \lambda+(1+\lambda)^{2}+\theta^{2} \beta^{2}}$ is the unique asymptotically stable equilibrium.

Proof. To verify that $q^{\star}=\frac{\theta^{2}}{\theta^{2}+\lambda}$ and $p^{\star}=\frac{\theta^{2} \lambda+(1+\lambda)^{2}}{\theta^{2} \lambda+(1+\lambda)^{2}+\theta^{2} \beta^{2}}$ are asymptotically stable, we first compute the Jacobian of the differential Equations (3) and (4). After simplifying, the Jacobian is:

$$
J(q, p)=\left[\begin{array}{cc}
p V\left(3 \theta^{2} q^{2}-4 \theta^{2}+\theta^{2}\right)-\frac{\lambda p V\left(2 q-3 q^{2}\right)}{(1+\lambda)^{2}} & \frac{q v(q-1)\left(\lambda q-\theta^{2}+q \theta^{2}\right)}{(1+\lambda)^{2}} \\
\frac{-p V(p-1)^{2}-\lambda p V(p-1)\left(\beta^{2} p-2 p+2\right.}{(1+\lambda)^{2}} & V\left(3 p^{2}-4 p+1\right)+\frac{q V\left(2 \beta^{2} p-4 p-3 \beta^{2} p^{2}+3 p^{2}+1\right.}{(1+\lambda)^{2}}-\frac{q V\left(2 \beta^{2} p-8 p-3 \beta^{2} p^{2}+6 p^{2}+2\right.}{(1+\lambda)}
\end{array}\right] .
$$

Plugging in $q^{\star}=\frac{\theta^{2}}{\theta^{2}+\lambda}$ and $p^{\star}=\frac{\theta^{2} \lambda+(1+\lambda)^{2}}{\theta^{2} \lambda+(1+\lambda)^{2}+\theta^{2} \beta^{2}}$, we get:

$$
J\left(q^{\star}, p^{\star}\right)=\left[\begin{array}{cc}
\frac{-\lambda \theta^{2} V\left(2 \lambda+\lambda \theta^{2}+\lambda^{2}+1\right)}{\left(\lambda+\theta^{2}\right)(1+\lambda)^{2}\left(2 \lambda+\beta^{2} \theta^{2}+\lambda \theta^{2}+\lambda^{2}+1\right)} & 0 \\
\frac{-\beta^{4} \theta^{2} V\left(\lambda^{2}+2 \lambda^{2} \theta^{2}+\lambda \theta^{2}+2 \lambda \theta^{2}+\lambda+\theta^{2}\right)}{\left(2 \lambda+\beta^{2} \theta^{2}+\lambda \theta^{2}+\lambda^{2}+1\right)^{3}} & \frac{-\beta^{2} \lambda \theta^{2} V\left(2 \lambda+\lambda \theta^{2}+\lambda^{2}+1\right)}{\left(\lambda+\theta^{2}\right)(1+\lambda)^{2}\left(2 \lambda+\beta^{2} \theta^{2}+\lambda \theta^{2}+\lambda^{2}+1\right)}
\end{array}\right] .
$$

Solving for the two eigenvalues gives:

$$
\begin{gathered}
\Lambda_{1}=\frac{-\lambda \theta^{2} V\left(2 \lambda+\lambda \theta^{2}+\lambda^{2}+1\right)}{\left(\lambda^{2}+\theta^{2}\right)(1+\lambda)^{2}\left(2 \lambda+\beta^{2} \theta^{2}+\lambda \theta^{2}+\lambda^{2}+1\right)}, \\
\Lambda_{2}=\frac{-\lambda \beta^{2} V\left(2 \lambda+\lambda \theta^{2}+\lambda^{2}+1\right)}{\left(\lambda+\theta^{2}\right)(1+\lambda)^{2}\left(2 \lambda+\beta^{2} \theta^{2}+\lambda \theta^{2}+\lambda^{2}+1\right)} .
\end{gathered}
$$

Since $\lambda, V, \theta, \beta>0$, both $\Lambda_{1}$ and $\Lambda_{2}$ are negative. As a result, since both eigenvalues are negative and real, the equilibrium is a sink and is asymptotically stable.

\section{References}

1. Olken, B.A.; Barron, P. The simple economics of extortion: Evidence from trucking in aceh. J. Political Econ. 2009, 117, 417-452. [CrossRef]

2. Klitgaard, R.E. Controlling Corruption; University of California Press: Berkeley, CA, USA, 1988.

3. Svensson, J. Eight questions about corruption. J. Econ. Perspect. 2005, 19, 19-42. [CrossRef]

4. Hauk, E.; Saez-Marti, M. On the cultural transmission of corruption. J. Econ. Theory 2002, 107, $311-335$. [CrossRef]

5. Fisman, R.; Miguel, E. Corruption, norms, and legal enforcement: Evidence from diplomatic parking tickets. J. Political Econ. 2007, 115, 1020-1048. [CrossRef]

6. Cameron, L.; Chaudhuri, A.; Erkal, N.; Gangadharan, L. Propensities to engage in and punish corrupt behavior: Experimental evidence from Australia, India, Indonesia, and Singapore. J. Public Econ. 2009, 93, 843-851. [CrossRef]

7. Barr, A.; Serra, D. Corruption and culture: An experimental analysis. J. Public Econ. 2010, 94, 862-869. [CrossRef] 
8. Banuri, S.; Eckel, C. New Advances in Experimental Research on Corruption: Chapter 3-Experiments in Culture and Corruption: A Review; Emerald Group Publishing: Bingley, UK, 2012.

9. Gachter, S.; Schulz, J.F. Intrinsic honesty and the prevalence of rule violations across socieites. Nature 2016, 531, 496-499. [CrossRef] [PubMed]

10. Chaudhuri, A.; Paichayontvijit, T.; Sbai, E. The role of framing, inequity, and history in a corruption game: Some experimental evidence. Games 2016, 7, 13. [CrossRef]

11. Bisin, A.; Verdier, T. The economics of cultural transmission and the dynamics of preferences. J. Econ. Theory 2001, 97, 298-319. [CrossRef]

12. Montgomery, J. Intergenerational cultural transmission as an evolutionary game. Am. Econ. J. Microecon. 2010, 2, 115-136. [CrossRef]

13. Bisin, A.; Verdier, T. Handbook of Social Economics: Chapter 9-The Economics of Cultural Transmission and Socialization; Elsevier: North Holland, The Netherlands, 2011.

14. Gunnoe, M.L.; Moore, K.A. Predictors of religiosity among youth aged 17-22: A longitudinal study of the national survey of children. J. Sci. Study Relig. 2002, 41, 613-622. [CrossRef]

15. Dunn, J.S.; Kinney, D.A.; Hofferth, S.L. Parental ideologies and children's after-school activities. Am. Behav. Sci. 2003, 46, 1359-1386. [CrossRef]

16. Hassan, K.E.; Kahil, R. The effect of "living values: An educational program" on behavior and 193 attitudes of elementary students in a private school in lebanon. Early Child. Educ. J. 2005, 33, 81-90. [CrossRef]

17. Smith, J.M.; Price, G.R. The logic of animal conflict. Nature 1973, 246, 15-17. [CrossRef]

18. Smith, J.M. Evolution and the Theory of Games; Cambridge University Press: Cambridge, UK, 1982.

19. McElreath, R. Reputation and the evolution of conflict. J. Theor. Biol. 2003, 220, 345-357. [CrossRef] [PubMed]

20. Doebeli, M.; Hauert, C. Models of cooperation based on the prisoner's dilemma and the snowdrift game. Ecol. Lett. 2005, 8, 748-766. [CrossRef]

21. Gintis, H. The evolution of private property. J. Econ. Behav. Organ. 2007, 64, 1-16. [CrossRef]

22. McElreath, R.; Boyd, R. Mathematical Models of Social Evolution: A Guide for the Perplexed; University of Chicago Press: Chicago, IL, USA, 2008.

23. Bowles, S.; Choi, J.-K. Coevolution of farming and private property during the early holocene. Proc. Natl. Acad. Sci. USA 2013, 110, 8830-8835. [CrossRef] [PubMed]

24. Makowsky, M.D.; Smaldino, P.E. The evolution of power and the divergence of cooperative norms. J. Econ. Behav. Organ. 2016, 126, 75-88. [CrossRef]

25. Broom, M.; Ruxton, G.D. Evolutionary stable stealing: Game theory applied to keptoparasitism. Behav. Ecol. 1988, 9, 397-403. [CrossRef]

26. Vickery, W.L.; Giraldeau, L.-A.; Templeton, J.J.; Kramer, D.L.; Chapman, C.A. Producers, scroungers, and group foraging. Am. Nat. 1991, 137, 847-863. [CrossRef]

27. Broom, M.; Luther, R.M.; Ruxton, G.D. Resistance is useless? Extensions to the game theory of kleptoparasitism. Bull. Math. Biol. 2004, 66, 1645-1658. [CrossRef] [PubMed]

28. Cavalli-Sforza, L.L.; Feldman, M.W. Cultural Transmission and Evolution: A Quantitative Approach; Princeton University Press: Princeton, NJ, USA, 1981.

29. Boyd, R.; Richerson, P.J. Culture and the Evolutionary Process; University of Chicago Press: Chicago, IL, USA, 1988.

30. Henrich, J.; Gil-White, F.J. The evolution of prestige: Freely conferred deference as a mechanism for enhancing the benefits of cultural transmission. Evol. Hum. Behav. 2001, 22, 165-196. [CrossRef]

31. Laland, K.N. Social learning strategies. Anim. Learn. Behav. 2004, 32, 4-14. [CrossRef]

32. Efferson, C.; Lalive, R.; Richerson, P.J.; McElreath, R.; Lubell, M. Conformists and mavericks: The empirics of frequency dependent cultural transmission. Evol. Hum. Behav. 2008, 29, 56-64. [CrossRef]

33. Mesoudi, A.; Lycett, S.J. Random copying, frequency-dependent copying and cultural change. Evol. Hum. Behav. 2009, 30, 41-48. [CrossRef]

34. Rendell, L.; Boyd, R.; Cownden, D.; Enquist, M.; Erikkson, K.; Feldman, M.W.; Fogarty, L.; Ghirlanda, S.; Lillicrap, T.; Laland, K.N. Why copy others? insights from the social learning strategies tournament. Science 2010, 328, 208-213. [CrossRef] [PubMed]

35. Molleman, L.; Pen, I.; Weissing, F.J. Effects of conformism on the cultural evolution of social behavior. PLoS ONE 2013, 8, e68153. [CrossRef] [PubMed] 
36. Bisin, A.; Verdier, T. A model of cultural transmission, voting and political ideology. Eur. J. Political Econ. 2000, 16, 5-29. [CrossRef]

37. Bisin, A.; Verdier, T. On the cultural transmission of preferences for social status. J. Public Econ. 1998, 70, 75-97. [CrossRef]

38. Tabellini, G. The scope of cooperation: Values and incentives. Q. J. Econ. 2008, 123, 905-950. [CrossRef]

39. Calbuig, V.; Olcina, G. Cooperation and cultural transmission in a coordination game. J. Econ. Behav. Organ. 2009, 72, 188-201. [CrossRef]

40. Bisin, A.; Topa, G.; Verdier, T. Cooperation as a transmitted cultural trait. Ration. Soc. 2004, 16, 477-507. [CrossRef]

41. Tullock, G. Efficient Rent Seeking; Texas A\&M University Press, College Station: College Station, TX, USA, 1980.

42. Saez-Marti, M.; Sjogren, A. Peers and culture. Scand. J. Econ. 2008, 110, 73-92. [CrossRef]

43. Guiso, L.; Sapienza, P.; Zingales, L. Alfred marshall lecture social capital as good culture. J. Eur. Econ. Assoc. 2008, 6, 295-320. [CrossRef]

(c) 2018 by the author. Licensee MDPI, Basel, Switzerland. This article is an open access article distributed under the terms and conditions of the Creative Commons Attribution (CC BY) license (http:/ / creativecommons.org/licenses/by/4.0/). 